\title{
Cooperative Tools for Remote Learning
}

\author{
Ivan C.A. de Oliveira, José A. C. Neto, Mariana L. Amaral \& J.R. de A. \\ Amazonas \\ Communications and Signals Laboratory of the Telecommunications and Control Engineering \\ Department, Escola Politécnica of the University of Sao Paulo, Sao Paulo, Brazil. \\ icalcan@dglnet.com.br,andre@lcs.poli.usp.br,nana_lgm@hotmail.com,jra@lcs.poli.usp.br
}

\begin{abstract}
This work describes the development of a prototype CSCW environment for learning called CTELCS with two groupwares that aid in the cooperative work, one synchronous and one asynchronous. The asynchronous groupware has features of an information sharing tool and looks like a FAQ with additional characteristics. The synchronous groupware is a project tool that supports the work in group with chat, video and cooperative resolution of activities. This prototype uses methodological criteria for appropriate use of different kinds of media (text, audio and video), modelling of courses content and, besides, makes an end-to-end assured QoS for session, authentication control and users maintenance.
\end{abstract}

Key words: CSCW, Remote Continuous Training, QoS.

\section{INTRODUCTION}

In the global learning scenario it is currently observed a great research effort by academic institutions, companies and even users for remote training through the Internet. In that kind of training, people in different places and time can take courses using remote learning employing users and courses maintenance tools, communication tools and progress control tools that can facilitate their learning. Those tools work with several kinds of media (text, audio and video) with different end-to-end QoS (Quality of Service). QoS is important since each media has different characteristics of storage and transport. The available learning environment has tools that make use of the media without worrying, in most of the cases, about QoS, spending time and

The original version of this chapter was revised: The copyright line was incorrect. This has been corrected. The Erratum to this chapter is available at DOI: 10.1007/978-0-387-35616-7_23 
even desmotivating their use. It is also observed that pedagogic methodologies are used in remote courses without specific criteria of the media use and without a content modelling in the attempt to motivate and to hold the students' attention. Besides, it can combine an individual approach with group collaborative activities. CSCW (Computer Supported Cooperative Works) environments with some groupwares can be used to provide this kind of feature [1]. This paper's objectives are: i) to present the construction of a prototype CSCW environment addressed to learning with assured end-to-end QoS, called CTELCS (Collaborative Training Environment of the Laboratory of Communications and Signals); ii) to apply the methodology on the content modelling and media adaptation for a remote course with great scalability, totally transported over the Internet [2]. The prototype includes the modeled content and two groupwares of a CSCW environment, one asynchronous and one synchronous. The asynchronous module is a collaborative application that looks like a FAQ (Frequently Asked Questions) with some additional functionality not found in applications of this kind, for example, searching and insertion of questions and answers concerning a given subject. The creation of these characteristics is facilitated by the use of XML (eXtensible Markup Language). The synchronous module is a project tool, that makes possible the work in group through chat, video and the unified solution of a problem. This application uses Java Applets and objects distributed with RMI (Remote Method Invocation) reducing the change of information between clients and applications server. The content generation, of the asynchronous module and the call of the synchronous module is accomplished through an applications server using Servlets.

The prototype is inserted in a distributed architecture for administration and control of packets switched network, called HCRM (Hierarchical Communication Resources Manager), that offers assured end-to-end QoS for different multimedia content distribution, access control and users authentication [3]. As CTELCS is inserted in this architecture, it serves as a platform of tests for HCRM and makes possible to offer all these functions to their users.

This paper is organized as follows: after this briefing introduction, section 2 introduces CSCW environments and their classifications, Interfacehuman-computer (IHC) and its importance and a discussion on existing technologies and which were chosen for the development of CTELCS; section 3 describes the methodology to be applied in the media adaptation and elaboration of remote learning contents; section 4 presents a description of the HCRM; section 5 discusses the CTELCS results and section 6 concludes paper and proposes some future works. 


\section{CSCW AND LEARNING ENVIRONMENTS, IHC AND TECHNOLOGIES: CURRENT STATE}

\subsection{CSCW and Learning Environments}

Learning environments are interesting when they combine the best among the support to the individualized study and the collaborative learning. A set of groupwares using different media can be built to form a typical $\mathrm{CSCW}$ environment, as email, chat, whiteboards and videoconference, with the objective of providing the collaborative learning. CSCW is a study area interested in the work in people groups with the help of computers [4]. The taxonomy most used in the classification of these environments takes into account: interaction way, geographical distribution and the types of the users applications (groupwares) according to Fig. 1.

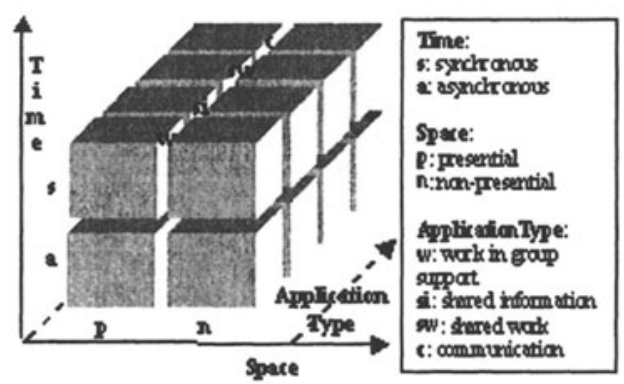

Figure 1. Graph of the groupwares classification in CSCW environments.

From the technological point of view, the collaboration among users depends on two tasks: i) the definition of a group and ii) the establishment (synchronous or asynchronous) of communication sessions. Another point equally important that has to be treated by collaborative applications is the coordination of the interdependencies among the activities that can be accomplished by the group [1]. Some known CSCW environments are BSCW (Basic Support for Cooperative Works), JETS (Java-Enabled Telecollaboration System) and JASMINE (Java Application Sharing in Multiuser Interactive Environments) [5][6][7][8]. A learning environment, globally used, that possesses some collaborative tools like chat and whiteboard is WebCT (World-Wide-Web Course Tools) [9].

A most important differential aspect of CTELCS is the assured end-toend QoS offered to the users. 


\subsection{IHC (Interface Human Computer)}

A fundamental part in the development of applications is the interface human computer, because through it the users have access to the functions of the system. In particular for learning applications, factors as motivation, satisfaction, efficiency, safety, training cost, return of the investment and learning efficacy depend, besides on the content quality and appropriate media use, on a good design interface. IHC involves several areas as Engineering, Design, Psychology and Linguistics.

The Fig. 2 illustrates the interaction process involved between the user and the system interface.

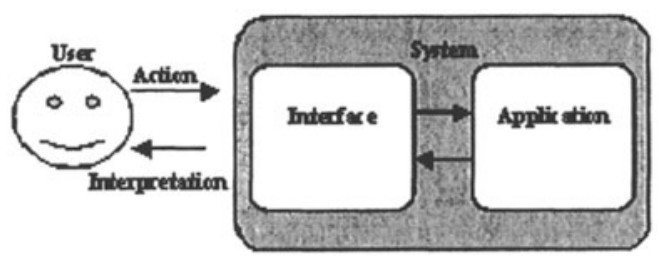

Figure 2. Interaction Process IHC

In learning applications several interaction styles can be adopted, as natural language, command language, menus, forms, WIMP(Windows, Icons, Menus and Pointers) and virtual reality. The closer the interface is to the student's reality, the learning and the motivation will be easier to be achieved.

The analysis of usability and communication characteristics in IHC is important, for this, heuristic and semiotics evaluations are applied [10].

The process of the design of interfaces can be driven independently of the implementation of the software, following a cyclical model that involves the specification of the needs, a prototype and evaluation. In the set of CTELCS applications an analysis of the requirements was accomplished and an interface was developed and then analysed by members of the research laboratory.

\subsection{Technologies}

The development of learning applications over the Internet can be accomplished using several kinds of technologies: static pages with pure HTML, powerful dynamic applications with CGI (Common Gateway Interface) and Servlets (Dynamic WebPages in Java) on the server side and Java Applets and JavaScript/CSS (Cascading Styles Sheets) on the client side that allow to modify or to adapt the content to be presented to the 
student depending on their actions; XML / XSL(eXtensible StyleSheet Language) that separates the content of the presentation and tends to be a standard for the Internet can be used too.

Any language can be used in the creation of CGI programs since the format allows to generate an executable. Examples of languages are PHP (Personal Home Page) and Perl (Practical Extraction and Report Language). Despite of the transparency and information uniformity search through the Web, CGI has inefficiency problems caused by the high overhead in servers with a lot of access, since to each request a copy of the program is shot. Java Servlets is an alternative and efficient format to CGI. A Servlet is a Java class that implements an analysis interface, execution and client answer. As being Java, Servlets make use of their advantages as portability, power, capacity and productivity and present better performance than CGI because they use threads for processing of simultaneous requests and the exclusion of interpreted languages [11].

Java Applets can be executed in the client machine starting from his request. It has advantages as creation of more complex interfaces, platform independence, no installation and updating need for the student, besides being removed after the end of its processing. There are some restrictions of safety that forbid to read or to write files in the local disk and the communication is restricted only to the server Applet origin. To turn around the communication restriction it can be implemented an scheme of requests repetition and messages exchange between server and the destiny of the message. It can use the platform of distributed objects RMI (Remote Method Invocation) in an attempt to reducing the communication between the clients and the server Applet origin [12].

JavaScript is an interpreted language, similar to Java and based on objects, that associated to CSS makes possible to develop dynamic pages with larger interaction allowing the creation of more attractive interfaces that can motivate the learning and to hold the student's attention [13].

$\mathrm{XML}$ is a metalanguage for the creation of new markup languages, defining the form of describing the information for a class of documents. The definition of the "content" type and of the markups is made using a DTD (Document Type Definition). The presentation format of data is accomplished using CSS or XSL. Currently there are several market formats, created by XML, some examples are, SMIL (Synchronized Multimedia Integration Language), XHTML (Extensible Hypertext Markup Language) and the open specification IMS Global Learning Consortium, Inc. (IMS) that seek to facilitate the activities of distributed on-line learning [13].

The CTELCS development and their groupwares are based on Servlets, HTML and JavaScript/CSS to make available the course content, Servlets 
and XML/XSL for the FAQ module and Applet/RMI in the creation of the collaborative project tool.

The chosen database server was MySql for possessing support of a great number of platforms, multiple languages, reliability, speed and access control [11].

\section{MEDIA AND CONTENT MODELLING:A METHODOLOGY}

\subsection{Introduction}

In the presential education scenario a lot of pedagogic theories are studied and applied, going from the behaviorism of Skinner, through the Interactive Constructivism of Piaget to the Social-Interactive Constructivism of Vygotsky. Remote training tries to make use of these pedagogic concepts but described how to appropriately use the media and how to model the content of the courses in an attempt to motivate and to hold the students attention contributing to earnings in the learning process are not described. Ref. [2] describes a methodology research that focuses mainly on the use of media and the content modelling for a remote course [1]. In this section some ideas of this methodology that were used in the CTELCS construction are exposed.

\subsection{The Media: Text, Audio and Video}

The text is the most common way for learning. There is the possibility to use different fonts types, sizes and colors that can help or to damage the cognition process depending on how it is applied. The effect of inserting appropriate illustrations in specific points of the text can be positive, allowing the learner to understand better and to easier remind the text theme. This media allows a great variety of transmission rates and in some situations, depending on the application, there is a need of real time transmission.

The audio is a media that does not demand broadband networks for transmission but the real time transport requires a bounded delay for the maintenance of the conversations. Audio of short duration, when inserted in strategic points, is appropriate to motivate, to discuss or to question the student. 
The video is characterized by a great volume of data and the need of bounded delay and synchronism for real time transmission. So, in technical terms, it is a media with high production, storage and transport cost. For teaching/learning process, however, the video can be much more interesting and when combined with audio trends to hold more the student's attention. The video finds competition only in the presence of games and simulators. Despite of the didactic-pedagogic advantages, due to the technical difficulties, videos should be reserved to explain concepts of difficult understanding.

\subsection{Content Modelling}

The programmatic content of a course is usually divided in modules. These modules can be further divided in smaller sub-modules because the human brain learns better in shorter stages.

To inform in a complete and immediate way the student's progress during the course has a motivation effect and it allows the student to direct his effort to points not assimilated yet. The information of this progress can be accomplished through the availability of successes and mistakes in exercises, self-tests, simulations and games strategically included in the modules/submodules.

\section{THE HIERARCHICAL RESOURCE COMMUNICATIONS MANAGER (HCRM)}

The HCRM can be seen as a proprietary protocol in the session layer of the OSI model, as shown in Fig. 3 [3]. It offers some services to the applications, as: establishment and session maintenance with QoS; users admission and dynamics metric evaluation and control of QoS. These aspects contribute to offer end-to-end QoS to HCRM applications.

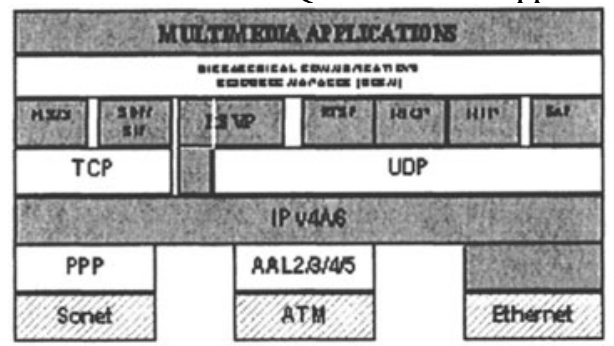

Figure 3. HCRM and protocols stack 
HCRM uses the SIP (Session Initial Protocol) protocol for the signalling among their components. An application can obtain information about the network QoS metric using the method MEASURE, an extension of SIP. The main attributes associated to that new method are:

- ipproxy: IP address of the proxy/router.

- idapp: application identifier. This identifier is obtained in the beginning of the application execution.

- idparam: it is the code of the requested parameter, examples are: QoS application, available bandwidth and full characterization.

The information obtained by the method MEASURE are useful to maintain the application aware of the network's QoS status. This characteristic allows the application to automatically adapts to changes of current available services end-to-end QoS.

The HCRM architecture illustrated in Fig. 4 has a proxy/router HCRM and a database in each local network with local users' information. The authentication and user validation are accomplished by consulting the local database. The database also keeps information about which services and media types are available for an user that accesses any application present in its architecture. Outside the local networks there is a central server and applications database where the CTELCS is located.

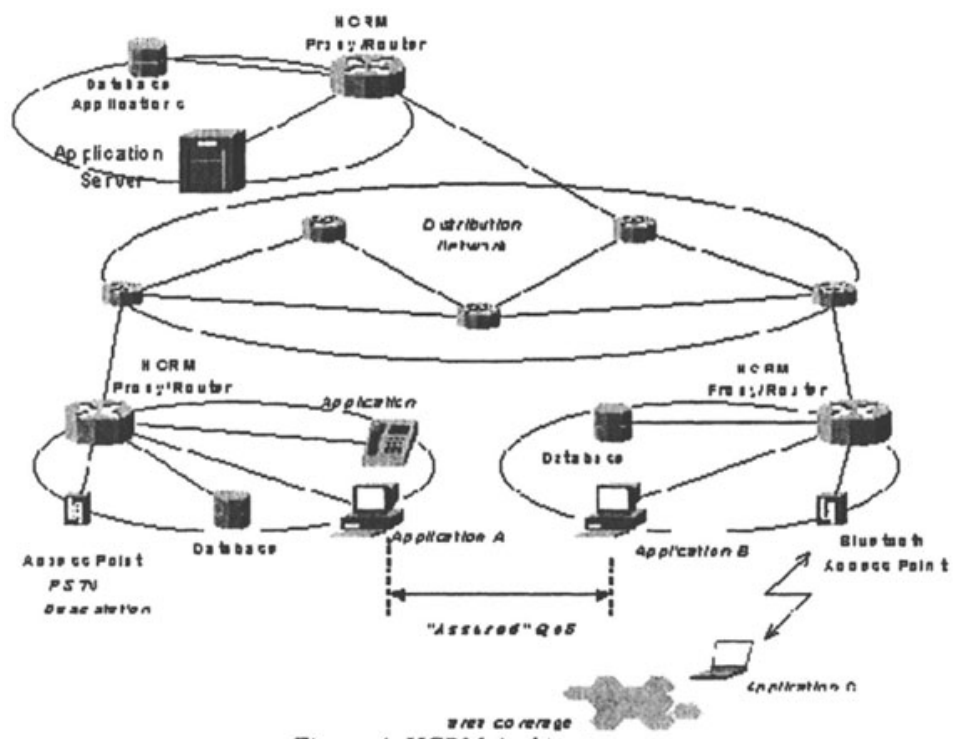

Figure 4. HCRM Architecture 
No matter where the CTELCS is located, the HCRM environment assures the accessibility to it (and any other application) given that it implements the RAS (Registration, Authentication, Status) function.

The user registered in the HCRM environment can access any multimedia application existent in the applications database, since he has rights for such access. When selecting CTELCS, the user enters in the learning application. Whenever a new on-line user enters the system there is an exchange of information between CTELCS and HCRM for obtaining user's data. These data maybe inserted, by CTELCS, in a list of users shared by all that are using it in the moment. This resource is interesting because it reduces the exchange of messages between CTELCS and the HCRM proxy/router, otherwise, the HCRM must be in charge of providing such functionality.

\section{PROPOSALS AND RESULTS}

\subsection{The Collaborative Training Environment of the Laboratory of Communications and Signals (CTELCS)}

The proposal of the CTELCS prototype is to be an environment of collaborative learning for training through the Internet with high scalability and with assured end-to-end session QoS. To achieve such feature CTELCS runs over the distributed architecture HCRM.

The CTELCS objectives are:

i) To provide the users' collaboration with the intention of accomplishing a better learning through:

- an asynchronous application where the student can send questions based in pre-defined subjects, in the moment of content study, to consult questions previously available, answered or not answered, and to answer some question.

- A collaborative synchronous tool for projects elaboration.

ii) To test the users control and QoS for established sessions offered by the HCRM.

To help the development of CTELCS, a typical example of a graduate course was selected, whose content is totally available and used for presential classes. 


\subsection{CTELCS Modules}

According to Fig. 5, CTELCS is a prototype that can be subdivided in three different modules.

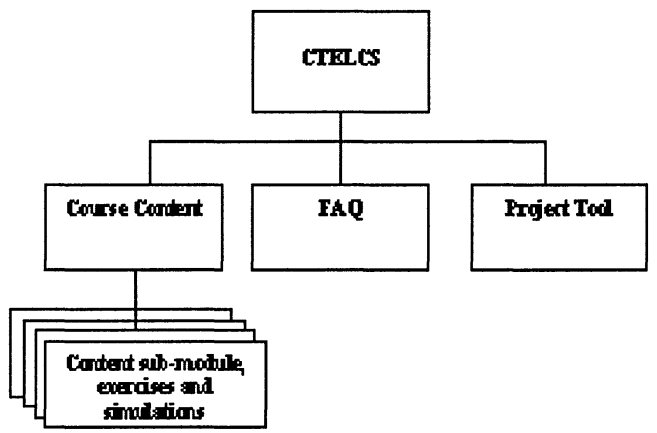

Figure 5. CTELCS modules

In Fig. 5, it can be observed that the content module of the course is divided in sub-modules containing exercises and simulations. The figure also illustrates two groupwares, a FAQ and a Project Tool. Classifying them according to the Fig. 1, it is verified that FAQ is asynchronous and nonpresential containing shared information and the Project Tool is synchronous and non-presential providing the work in group support. Fig. 6 illustrates this classification.

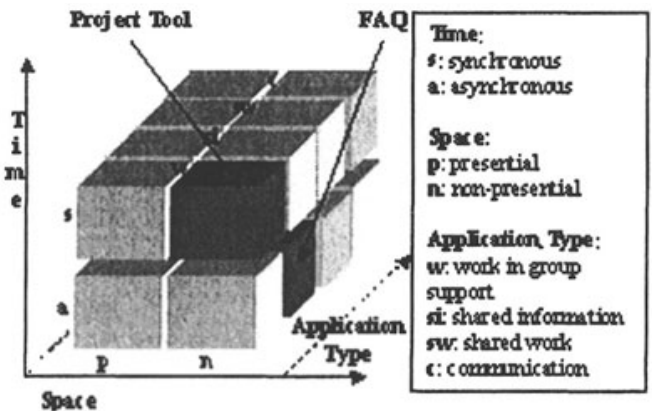

Figure 6. Graph of CTELCS modules classification

\subsubsection{Modelling of the Course Content}

In the modelling of the content interface, usability and communication criteria were analysed trying to create a more pleasant and motivating environment [10]. According to the methodology described in section 3 , the 
content of the selected graduate course material was subdivided in small modules, without any significant modification in the text, some appropriate media were added to the text, as image and/or animation. In each submodule was verified the possibility of introducing simulations, the creation of evaluations like self-test and more elaborated exercises to inform the student's progress. Examples of the content interface can be visualized in the Fig. 7.

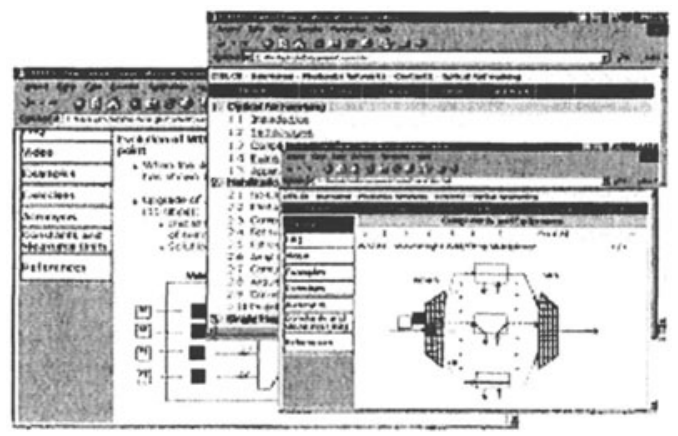

Figure 7. Example of the content interface in CTELCS.

The process of Interface generation is obtained through the user request on a link of a HTML page. This request is sent to Servlet in the applications server that treats the request and returns the resulting page. The pages possess JavaScript/CSS as a form of trying to improve navigation aspects and the user interaction with the content as shown in Fig. 8.

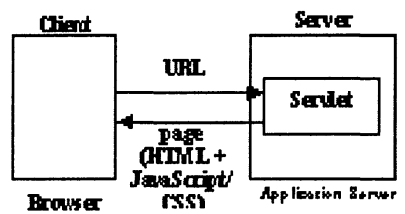

Figure 8. Content interfaces generation process.

\subsubsection{FAQ Modelling}

The FAQ's proposal is the construction of an asynchronous nonpresential tool, where the student can formulate and answer doubts in the moment of the study of some topic of the course. The answers to the doubts can be performed in a cooperative way by any of the participants. It is important to observe that this module differs of known FAQs by allowing the student/teacher to insert associated subjects to the question/answer. 
Fig. 9 shows that its structure follows a three-tier architecture, using $\mathrm{XML} / \mathrm{XSL}$ to accomplish the creation of the document with the questions/answers requested obtained from the database.

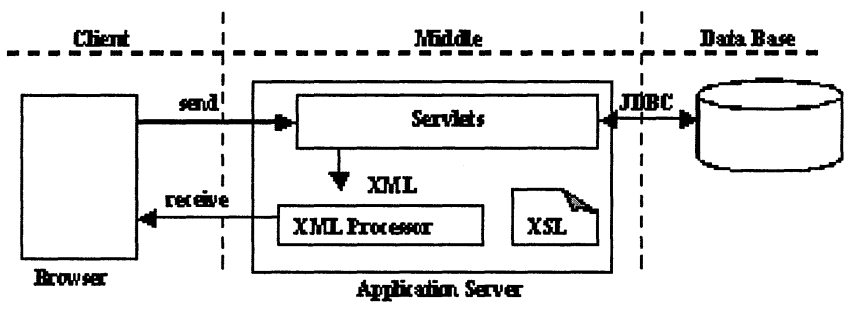

Figure 9. Three-tier architecture.

According to Fig. 9, the user makes a request (send). The Servlet obtains the data from the database setting up the XML document. This is processed and joined to correspondent XSL and returned to the client that visualizes the content (receive). Fig. 10 illustrates a simplified part of DTD and XML document .

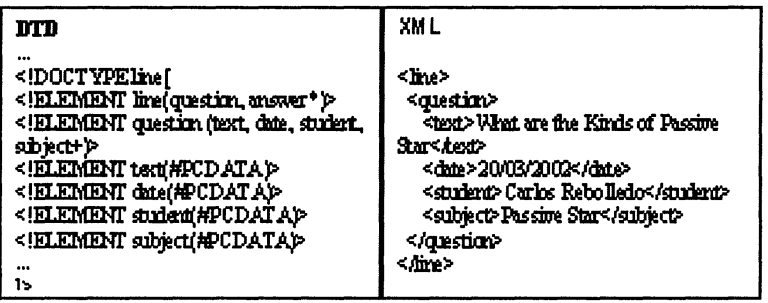

Figure 10. Simplified example of a DTD and a XML documents for the FAQ.

Thus, the FAQ is a collaborative asynchronous tool with information sharing. Teacher and student can interact with the tool. There is a differentiated interface for both teacher and student allowing a larger control on the part of the teacher, as shown in Fig. 11. 


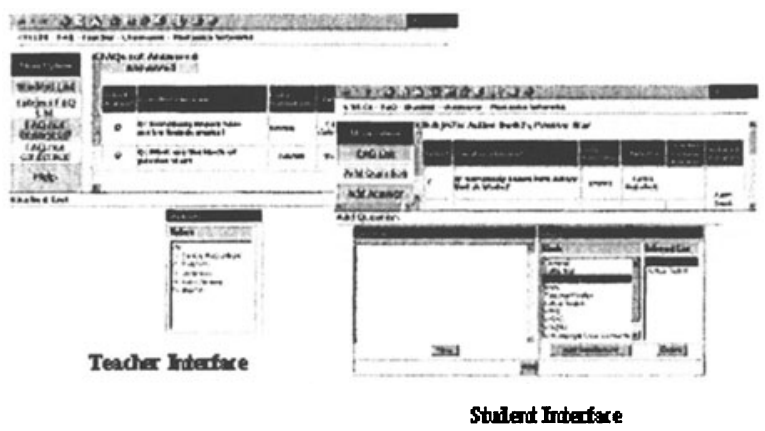

Figure 11. FAQs interface models.

The student's options are:

- FAQ List: selects the related subject with his doubt and all the answered questions or not.

- Add Question: writes the question about a certain subject. In case that no subject is selected the question is considered as general.

- Add Answer: answers questions previously selected in the FAQ list.

- Add Subject: inserts some subject so far non-existing in the subjects list.

- Help: help online about how to use the tool.

All answers can be analysed by the teacher that can confirm or correct them.

For the teacher, besides the same items as to the student, there is an option of visualizing all questions answered by the students. This option is interesting, given that the teacher can verify the student's evolution, with the possibility to do analysis of the number of answered questions, in correct or wrong way, and the number of questions made.

An UML (Unified Modelling Language) modelling simplified of the FAQ data stored in the database server is illustrated in the Fig. 12, involving the classes: Question, Answer and Subject. 


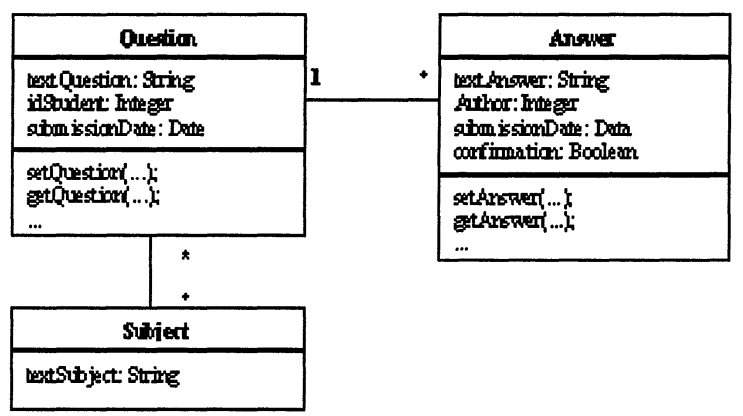

Figure 12. Classes simplified for FAQ in UML.

\subsubsection{Modelling of the Project Tool}

The projects tool is a synchronous non-presential groupware that makes possible the cooperative work in the solution of the course projects, for a group of previously defined people. It is worth to emphasize that project tools are interesting for the learning process, mainly when they deal with subjects with high complexity degree. Otherwise, they are relatively dependent on the kind of the course.

The teacher's basic functions are:

- To describe projects and the maximum period of time for delivering the solution;

- To set up the students' groups. This solution was adopted in an attempt to reduce the complexity of the problem. More complex and interesting solutions will be implemented later with the technology of Intelligent Agents for the automatic creation of students' groups [1];

- To visualize the results of the elaborated projects and sending his analysis to the group by e-mail.

The group's activities are comprised of:

- To mark the synchronous activity by e-mail among all the participants;

- To read the information of the proposed project;

- To elaborate the solution of the problem. During the process of the solution construction, messages are exchanged among the participants, via chat and/or video resources attached to the tool. These additional resources make possible the cooperative work.

- To save the solution or set of solutions obtained by the group.

The treatment of the interdependence of the group activities is treated in a simple way. Only one of the members of the group can work on the collaborative interface, assuming the role of activities coordinator. The other 
members, denominated collaborators, will be suggesting solution approaches and visualizing the result. The role change among the participants is a coordinator's responsibility. The change can be proposed by the coordinator or accepted by him/her according to a previous request.

The proposal of the tool implementation as an Applet avoids the installation need of some application in the client's machine and makes it possible the creation of a more elaborated interface. In this Applet is also interesting the use of objects distributed via RMI, aiming to reduce the amount of information exchanged between the applications server and the Applet. The use of distributed objects makes it possible to the Applet exchange messages with the server only for students on-line list updating and to verify if the users that have entered in the project tool are active or not.

In the example of the Fig. 13 is observed the itinerary of the Applet to the students, symbolized by an envelope, for a group of 3 students. To the group's student that first loads the Applet (Student A) is allocated the coordinator role. The other members of the group (Students B and C) will be collaborators. The coordinator and the collaborators should get the project proposed by the teacher, beginning in an effective way the collaboration on the problem solution.

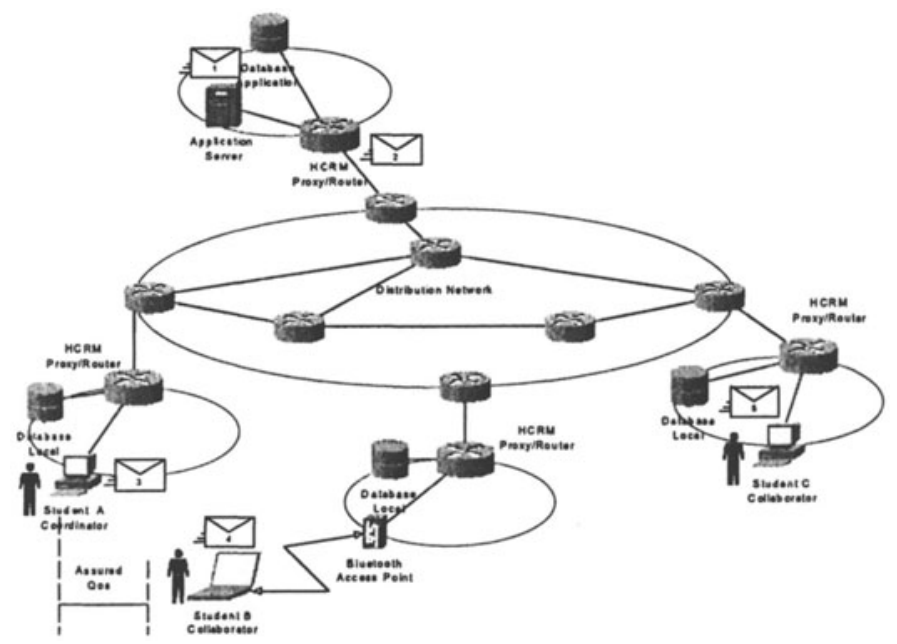

Figure 13. Process of Applet load and collaboration among the 3 participants of a group in the HCRM environment. 
A sketch of the application interface seen by the student follows the schema of Fig. 14.

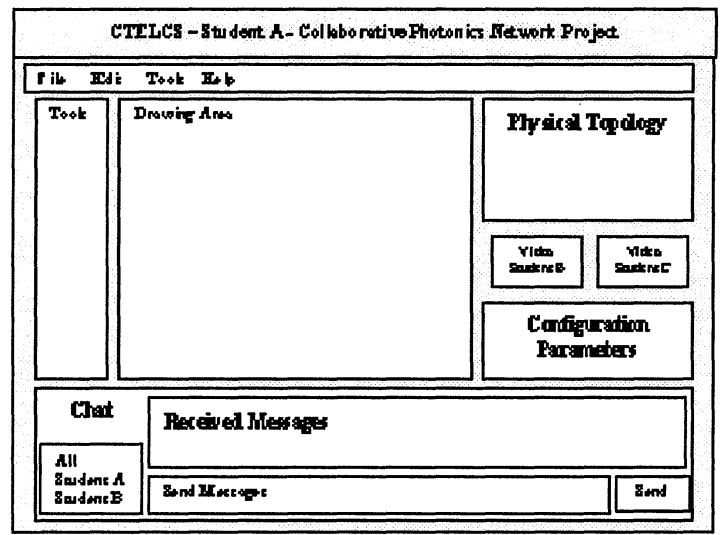

Figure 14. Sketch of the Student Interface for the Projects Tool.

\section{CONCLUSIONS AND FUTURE WORKS}

The usability criteria and the methodology described in the section 3 applied to the interface and to the content of the chosen course allowed to model the interface and the content to obtain a good navigability, good structure and adaptation of the media. The insertion of CTELCS in the HCRM architecture frees the application of the programming effort for assuring end-to-end QoS, authentication, registration, control and maintenance of session users. The linking of all mentioned characteristics certainly are motivating and an attraction factor to CTELCS users.

The asynchronous FAQ module allows to classify and to look for questions and answers about different subjects, besides making possible to the teacher to perform analysis about the students usage of the available FAQ tools. The use of objects distributed via RMI in the projects tool reduces the amount of exchanged information changed clients and server, therefore increasing the system performance. The use of these groupwares makes possible a larger interaction among those involved in the collaboration, improving the individual learning. CTELCS also implements an on-line list of students sharing in the applications server and thus minimizing the flow of messages between HCRM and CTELCS.

Future works include:

- Mechanisms of automatic creation of students groups using the Intelligent Agents technology . 
- A more interesting schema of treatment of the interdependencies the collaborative tasks.

- Simulations that make use of virtual reality in VRML and/or Java 3D, approximating the student of the real world.

- Implementation of the standardization IMS in the content module.

\section{AKNOWLEDGMENTS}

Ericsson Telecommunications supported this work under the contract Ericsson/FDTE-EPUSP No.TC-790.

\section{REFERENCES}

[1] L. P. Magalhães, "Final report of SAPIENS Project Activities," UNICAMP/USP,2001.Available:http://www.dca.fee.unicamp.br/projects/sapiens/Reports/ rf2000/rf2000.html.

[2] J. A. Caruso, I. C. A. Oliveira, M. L. Amaral, J. R. A. Amazonas, "Learning Through Telecomputing," submitted to 2002 IFIP Workshop on Internet Technologies, Applications, and Societal Impact, Poland, October, 2002. Available: icalcan@dglnet.com.br.

[3] J. P. Costa, A. B. Lima, C. R. Barra, J. R. A. Amazonas, "An Environment for Management and Control of End-to-End QoS Over IP-Based Heterogeneous Networks," submitted to IFIP/IEEE International Conference on Multimedia Networks and Services Managing IP Multimedia End-to-End, USA, October, 2002.

[4] A. B. Raposo, L. P. Magalhães, L. M. Ricarte, "Interação na WEB," Proc. XIX National Conference of the Brazilian Society of Computer Science, Rio de Janeiro, Brazil, 1999.

[5] P. Jefferies, I. Constable, "Using BSCW in Learning \& Teaching," Proc. of Educational Technology \& Society, 2000. Available: http://ifets.ieee.org/periodical/vol_3_2000/b03.html.

[6] S. Shirmohammadi and N.D. Georganas, "JETS: Java-Enabled TeleCollaboration System," Proc.IEEE Multimedia Systems'97, Ottawa, June 1997. Available: http://www.mcrlab.uottawa.ca/papers/Shirmohammadi97IEEE.ps.gz.

[7] A. El Saddik, S.Shirmohammadi, N.D.Georganas, R.Steinmetz, "JASMINE: Java Application Sharing in Multiuser INteractive Environmnets," Proc. 7th International Workshop on Interactive Distributed Multimedia Systems and Telecommunication Services (IDMS2000), CTIT / University of Twente, Enschede, The Netherlands, October 2000. Available: http://www.mcrlab.uottawa.ca/papers/jasmine-idms2000.pdf.

[8] S. Shirmohammadi , J.C. de Oliveira,, N. D. Georganas, "Applet-Based Telecollaboration: A Network-centric Approach," IEEE Multimedia, pags. 64-73, vol. 5 April-June 1998. Available: http://www.mcrlab.uottawa.ca/ jauvane/papers/JETS-IEEE-MMM.pdf.

[9] S. Hazari, "Evaluation and Selection of Web Course Management Tools,". University of Maryland, College Park, June, 1998. Available: http://sunil.umd.edu/webct/.

[10] J. Nielsen, "Designing Web Usability: The Practice of Simplicity," New Riders Publishing, USA, 2000. Available: http://www.useit.com/jakob/webusability/.

[11] D. Harms, "JSP, Servlets, and MySQL," M\&T Books, New York, NY, 2001. 
272 Ivan C.A. de Oliveira, José A. C. Neto, Mariana L. Amaral \& J.R. de A. Amazonas

[12] T. B. Downing, "Java ${ }^{T M}$ RMI: Remote Method Invocation," IDG Books WorldWide, Foster City, CA, 1998.

[13] H. M. Deitel, P. J. Deitel, T. R. Nieto, T. M. Lin, P. Sadhu, "XML How To Program," Prentice-Hall, Inc, New Jersey, 2001. 